\title{
Correction to: Amphi-enterobactin commonly produced among Vibrio campbellii and Vibrio harveyi strains can be taken up by a novel outer membrane protein FapA that also can transport canonical $\mathrm{Fe}(\mathrm{III})$-enterobactin
}

\author{
Hiroaki Naka ${ }^{1} \cdot$ Zachary L. Reitz $^{2} \cdot$ Aneta M. Jelowicki $^{2} \cdot$ Alison Butler $^{2} \cdot$ Margo G. Haygood $^{1}$ \\ Published online: 5 October 2018 \\ (c) SBIC 2018

\section{Correction to:} \\ JBIC Journal of Biological Inorganic Chemistry \\ https://doi.org/10.1007/s00775-018-1601-5
}

In the original publication, third author's name was incorrectly published as Aneta L. Jelowicki.

The correct name should read as Aneta M. Jelowicki.

The original article can be found online at https://doi.org/10.1007/ s00775-018-1601-5.

Margo G. Haygood

margo.haygood@utah.edu

1 Department of Medicinal Chemistry, L.S. Skaggs Pharmacy Institute, University of Utah, Salt Lake City, UT, USA

2 Department of Chemistry and Biochemistry, University of California, Santa Barbara, USA 